\title{
Analysis and design of different controllers for non-linear power systems
}

\author{
Rekha*, A. K. Singh \\ Asst. Professor, Electrical Engg. Dept., NIT Jamshedpur \\ Professor, Electrical Engg. Dept., NIT Jamshedpur \\ *Corresponding author E-mail: rchy72@gmail.com
}

\begin{abstract}
The objective of this paper is to design controller for non-linear power system using Direct Feedback Linearization technique to improve the transient stability and to achieve better voltage regulation. In case of fault in the power system, power angle and the terminal voltage are the parameters which are to be monitored. The simulation has been carried out taking different values of initial power angles and results were obtained for power angle and terminal voltage. To overcome the demerits of DFL-LQ optimal controller and DFL voltage regulator, co-ordinated controller is proposed. Simulation results show that transient stability of a power system under a large sudden fault has been improved by using co-ordinated controller.
\end{abstract}

Keywords: Stabilizer, FLC (fuzzy logic controller), DFL (direct feedback linearization), Co-ordinated controller

\section{Introduction}

The power system dynamics stability field is an ever expanding area of research due to large scale interconnection of power systems. This area [1] has been acknowledged as an important problem for secure system operation. Non-linear control theory is considered here to design controllers for power systems to improve the transient stability and to achieve voltage regulation. For engineering systems, large range of operations are there and the non-linearities and uncertainty involved in the system models [2], [3] as well as disturbances present in the environment, cause the extreme complexity of the global control problem. A general global control structure is based on the qualitative analysis of the dynamical systems. Local controllers are designed based on local models and local performance requirements and coordination rules are employed to combine [4], [5] the local elements control.

The problem of designing controller to prevent an electric power system losing synchronism after a large sudden fault is of great importance in power system design [6]. In recent years, most of the non-linear excitation controllers have been developed based on the classical third order dynamic generator model [7]. The simulation results showed that such a simplification has very little effect on the performances of the designed controllers. Establishing a global control structure for general non-linear control systems [8] is preferred, as control of complex system over a wide range of operating conditions can be achieved for a set of control objective. When the parameters in the power system are known a DFL control law is developed to linearize the plant. Whereas, when a large sudden fault occurs, the reactance of the transmission line changes a lot. For this type of case robust controller design approach [13], [14] is used. The simulations are done by taking different initial power angle and results were obtained for power angle and terminal voltage.

\section{Non-linear systems}

Non-linear control has attracted particular attention during the past few decades as virtually all physical systems are non-linear [5] in nature. Non-linear control [12] analysis and design provides a sharper understanding of real world. For real world systems, large ranges of operation cannot be avoided, and demanding specifications are required by modern technology such as high-performance robotics and aircraft which typically involve non-linear dynamics. 
Control of complex systems has emerged as an extremely important topic in the past few years. Control cannot be realized without a good understanding of the system structure and behavior. In most practical situations, a system operates in several different conditions and co-ordinates different control goals. In power system, the disturbance can be small in the form of load changes taking place continually, or a large like a severe short circuit on the transmission line. Uncertainities mainly relate to system structure and loads [11]; some important parameters like the reactances of the transmission lines are not constant due to a variety of effects such as lighting outages, severe storms, equipment failures etc. the fault considered in this paper is 3-phase short circuit fault occurring on the transmission line.

\section{PID Controllers}

Classical and modern methodologies in linear and non-linear control provide powerful design tools for systems modeled by ordinary differential equation. However, linear methods are valid for a small operating region, and many non-linear methods are only effective within a certain operating region due to the non-existence of global geometric structures. Multiple controllers are widely used in practice where controllers adapt themselves to different operating conditions and are able to co-ordinate various control requirements. Control of a complex system over a wide range of operating conditions to achieve a set of control objectives is called global control. To achieve the goals of global control, multiple controllers are needed. They are derived from the design method of local control and operate over different operating points during different time periods to fulfill the corresponding primary control requirements. Therefore how to coordinate the controllers is the major issue of global control. A plant model of a physical system that is to be controlled is usually very complex and difficult. Adaptive control is a model-free controller that can be used to control non-linear systems. Most of the adaptive controllers involve certain types of function approximator from input/output experiments. The basic objective of adaptive control is to maintain consistent performance of a control system in the presence of the designed parameters. Traditional adaptive controllers

\section{Controller design techniques}

Since power system are highly non-linear and the operating conditions can vary over a wide range, the conventional PSS (CPSS) performance is degraded when the operating point changes from one to another because of fixed parameters of stabilizers. Metaheuristic optimization technique like GA, Tabu search, simulated annealing, bacteria foraging, particle swarm optimization. [8], [10]

\subsection{Power system damping controller structure}

Power System Stabilizer model consists of:

1. Gain block

2. Washout block

3. Phase compensation block

The input to PSS is rotor speed deviation, output is auxiliary excitation signal given to the generator excitation system.

$\Delta \mathrm{U} / \Delta \mathrm{W}=K s\left(1+s T_{i}\right)\left(s T_{w}\right) /\left(1+s T_{2}\right)\left(1+s T_{w}\right)$

where

Ks - PSS gain

$T_{1}, \quad T_{2}$ - PSS time constant

Signal washout function is a high pass filter which removes DC signals. The washout time is in the range of 1-20 sec Eg. $T_{w}=15 \mathrm{sec}$

Eigen value based objective function

$[\downarrow]=\operatorname{Max}\{\operatorname{Re}[\lambda i]\}, \lambda i \varepsilon \lambda_{\tau}$

Where $\lambda i$ belongs to the group of electromechanical mode Eigen values $\lambda \tau$.

\subsection{Fuzzy controller based design}

FLC are a class of non-linear controllers that make use of human expert knowledge and an implicit impression to apply control to such systems. The construction of these controllers can be quick and effective in the presence of expert knowledge. In the absence of such knowledge, their design can be slow and based on trial and error rather than a guided approach. Genetic Algorithm provides a way of surmounting this shortcoming. These algorithms use some of the 
concepts of evolutionary theory, and provide an effective way of searching a large and complex solution space to give close to optimal solution in much faster time than random trial and error. Fuzzy control can be an especially effective way of controlling non-linear systems when expert human knowledge of the system is available. GA is fully capable [16] of creating complete fuzzy controller given the equation of system, eliminating the need for human input in the design loop.

Table 1: Comparisons of different control designs

\begin{tabular}{llll}
\hline & Work range & Simple & stability \\
\hline Linear & $(-\pi / 4 \pi / 4)$ & Yes & Local \\
Non-linear & $(-\pi / 2 \pi / 2)$ & No & Non-local \\
Fuzzy PDC & $(-\pi \pi)$ & No & Non-local \\
\hline
\end{tabular}

A properly designed voltage regulating system [12] can increase the steady state stability limit of a synchronous machine by a considerable amount.

Fast regulator and exciter action and low transient reactance are desirable. The amplification factor of the regulating system must be co-ordinated properly with the machine and system constants are made as large as possible.

Amortisseur windings have little effect on the possible gain in power limit. For maximum gain in power limit, a regulator with broad regulation and rapid response should be used.

\section{Mathematical modeling of the system}

Electrical power systems are complex multivariable dynamic systems. The simplified model [9] can be described as a single generator connected through two parallel transmission line to a very large network approximated by an infinite bus. The model is shown in fig. 1. The fault we consider in this paper is a symmetrical 3-phase short circuit which occurs on one of the transmission lines.

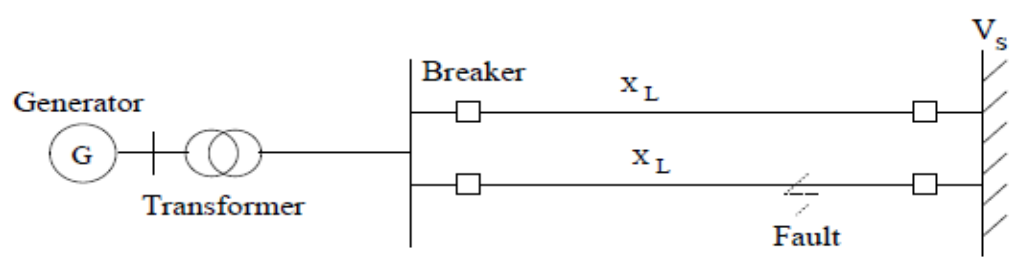

Fig. 1: Single machine-infinite bus model

The following abbreviations are used while designing the model:

$\Delta \delta(t)=\delta(t)-\delta_{0}$

$\delta(t)=$ power angle of the generator

$\delta_{0}=$ power angle of the generator at the operating point;

$\omega(\mathrm{t})=$ the relative speed of the generator

$\Delta P e=P e(t)-P m$

$P m=$ mechanical input power

$P e=$ active electric power delivered by the generator;

$\omega_{0}=$ synchronous machine speed; $\omega_{0}=2 \pi \mathrm{f}_{0}$

$\mathrm{D}=$ per unit damping constant

$\mathrm{H}=$ per unit inertia constant

$\mathrm{E}_{\mathrm{q}}(\mathrm{t})=$ the transient emf in the quadrature axis

$\mathrm{E}_{\mathrm{f}}(\mathrm{t})=$ the equivalent emf in the excitation coil;

$\mathrm{T}_{\mathrm{do}}=$ the direct axis transient short circuit time;

$\mathrm{Q}_{\mathrm{e}}(\mathrm{t})=$ the reactive power

$\mathrm{I}_{\mathrm{f}}(\mathrm{t})=$ the excitation current

$\mathrm{I}_{\mathrm{q}}(\mathrm{t})=$ the quadrature axis current;

$\mathrm{V}_{\mathrm{t}}(\mathrm{t})=$ the generator terminal voltage;

$\mathrm{K}_{\mathrm{c}}=$ the gain of the excitation amplifier;

$\mathrm{u}_{\mathrm{f}}(\mathrm{t})=$ the input of the SCR amplifier of the generator

$x_{d s}=x_{T}+\frac{1}{2} x_{L}+x_{d}$ 
$\hat{x}_{d s}^{\prime}=x_{T}+\frac{1}{2} x_{L}+x_{d}{ }^{\prime}$

$x_{T}=$ reactance of the transformer

$x_{d}=$ direct axis reactance

$x_{L}=$ the reactance of transmission line; $\quad x_{S}=x_{T}+\frac{1}{2} x_{L}$

$x_{e d}=$ the mutual reactance between the excitation coil and the stator coil;

$\mathrm{V}_{\mathrm{s}}=$ the infinite bus voltage;

Mechanical equation:

$\Delta \dot{\delta}(t)=\omega(t)$

$\dot{\omega(t)}=\frac{-D}{H} \omega(t)-\frac{\omega_{0}}{H} \Delta P_{e}(t)$

Generator Electrical Dynamics:

$\dot{E} \dot{q}(t)=\frac{1}{T_{d o}}\left(E_{f}(t)-E_{q}(t)\right)$

Electrical Equations:

$E_{q}(t)=\frac{x_{d s}}{x_{d s^{\prime}}} E_{q}^{\prime}(t)-\frac{x_{d}-x_{d^{\prime}}}{x_{d s}^{\prime}} V_{s} \cos \delta(t)$

$E_{f}(t)=K_{c} u_{f}(t)$

$P_{e}(t)=\frac{V_{s} E_{q}(t)}{x_{d s}} \sin \delta(t)$

$I_{q}(t)=\frac{V_{s}}{x_{d s}} \sin \delta(t)=\frac{P_{e}(t)}{x_{a d} I_{f}(t)}$

$Q_{e}(t)=\frac{V_{s}}{x_{d s}} E_{q}(t) \cos \delta(t)-\frac{V_{s}^{2}}{x_{d s}}$

$E_{q}(t)=x_{a d} I_{f}(t)$

$V(t)=\frac{1}{x_{d s}}\left\{x_{s}^{2} E_{q}^{2}(t)+V_{s}^{2} x_{d}^{2}+2 x_{s} x_{d} x_{d s} P_{e}(t) \cot \delta(t)\right\}^{1 / 2}$

\section{Non-linear controller design for the power system}

From the model discussed above, we know that the power system model is non-linear [12]. In this section we discuss the design principles using DFL technique to design non-linear controller for a power system.

Since $E_{q}^{\prime}(t)$ is physically un-measurable, we eliminate $E_{q}^{\prime}(t)$ by differentiating equation (6) and using (1) to (6)

Equation (6) is $P_{e}(t)=\frac{V_{s} E_{q}(t)}{x_{d s}} \sin \delta(t)$

$$
\begin{aligned}
& \frac{d P_{e}(t)}{d t}=\frac{V_{s}}{x_{d s}} E_{q}(t) \cos \delta(t) \dot{\delta}(t)+\frac{V_{s}}{x_{d s}} \dot{E}_{q}(t) \sin \delta(t) \\
= & \frac{V_{s}}{x_{d s}} E_{q}(t) \cos \delta(t) \omega(t)+\frac{V_{s}}{x_{d s}} \sin \delta(t)\left[\frac{x_{d s}}{x^{\prime} d s} \dot{E}_{q}^{\prime}(t)+\frac{x_{d}-x_{d}^{\prime}}{x_{d s}^{\prime}} V_{s} \sin \delta(t) \omega(t)\right] \\
= & \frac{V_{s}}{x_{d s}} E_{q}(t) \cos \delta(t) \omega(t)+\frac{V_{s}}{x_{d s}} \sin \delta(t)\left[\frac{x_{d s}}{x_{d s}^{\prime}} \frac{1}{T_{d o}}\left(E_{f}(t)-E_{q}(t)\right)+\frac{x_{d}-x_{d}^{\prime}}{x_{d s}^{\prime}} V_{s} \sin \delta(t) \omega(t)\right]
\end{aligned}
$$

We have $T_{d o}^{\prime}=\frac{x_{d s}}{x_{d s}} T_{d o}$

$$
\begin{gathered}
=-\frac{1}{T_{d o} o^{\prime}} P_{e}(t)+\frac{1}{T_{d o}}\left\{\frac{V_{s}}{x_{d s}} \sin \delta(t)\left[K_{c} u_{f}(t)+T_{d o}\left(x_{d}-x_{d}\right) \frac{V_{s}}{x_{d s}} \sin \delta(t) \omega(t)\right]+T_{d o}^{\prime} \frac{V_{s}}{x_{d s}} E_{q}(t) \cos \delta(t) \omega(t)\right\} \\
=-\frac{1}{T_{d o}}, \Delta P_{e}(t)+\frac{1}{T_{d o}},\left\{\begin{array}{c}
\frac{V_{s}}{x_{d s}} \sin \delta(t)\left[K_{c} u_{f}(t)+T_{d o}\left(x_{d}-x_{d}\right) \frac{V_{s}}{x_{d s}} \sin \delta(t) \omega(t)\right]+ \\
T_{d o}^{\prime} \frac{V_{s} E_{q}(t)}{x_{d s}} \cos \delta(t) \omega(t)-P_{m}
\end{array}\right\},
\end{gathered}
$$

As $\Delta P_{e}(t)=P_{e}(t)-P_{m}$

Therefore

$$
\Delta P_{e}^{\prime}(t)=-\frac{1}{T_{d o}} \Delta P_{e}(t)+\frac{1}{T_{d o}}, v_{f}(t)
$$

where $v_{f}(t)=\frac{V_{s} \sin \delta(t)}{x_{d s}}\left[K_{c} u_{f}(t)+T_{d o}\left(x_{d}-x_{d}\right) \frac{V_{s}}{x_{d s}} \sin \delta(t) \omega(t)\right]+T_{d o}^{\prime} \frac{V_{s} E_{q}(t)}{x_{d s}} \cos \delta(t) \omega(t)-P_{m}$ or, $v_{f}(t)=I_{q}(t)\left[K_{c} u_{f}(t)+T_{d o}\left(x_{d}-x_{d}\right) \frac{V_{s}}{x_{d s}} \sin \delta(t) \omega(t)\right] \quad+T_{d o}^{\prime}\left[Q_{e}(t)+\frac{V_{s}^{2}}{x_{d s}}\right] \omega(t)-P_{m}$

The model (1) to (3) is therefore linearized 
The linearized model is

$\Delta \dot{\delta}(t)=\omega(t)$

$\dot{\omega}(t)=\frac{-D}{H} \omega(t)-\frac{\omega_{0}}{H} \Delta P_{e}(t)$

$\Delta P_{e}^{\prime}(t)=-\frac{1}{T_{d o}{ }^{\prime}} \Delta P_{e}(t)+\frac{1}{T_{d o}{ }^{\prime}} v_{f}(t)$

where $v_{f}(t)$ is the new input.

When the parameters in the power system are known, we can design a DFL control law to linearize the plant. But when a large sudden fault occurs the reactance of the transmission line $\mathrm{x}_{\mathrm{L}}$ changes a lot. This change is treated as parametric uncertainty [13],[14]. After linearization, we can employ linear control theory, such as LQ-optimal control theory, to design a feedback law

$v_{f}(t)=f\left(\delta(t), \omega(t), P_{e}(t)\right)$

To give the desired stability and performance properties, $v_{f}(t)$ and $P_{e}(t)$ are the control inputs.

\section{Different controllers and their problems}

\subsection{DFL non-linear controller}

The feedback linearization is a quite appealing design method for non-linear systems. Since it avoids the local nature of approximate linearization and transforms the system to be linear over a very wide range.

$\dot{\mathbf{d}}=\mathbf{w}$

$\dot{w}=\frac{-D}{2 H} w-\frac{w_{0}}{2 H} \Delta P_{e}$

$\Delta \dot{P}_{e}=\frac{-1}{T^{\prime}} \Delta P_{e}-\frac{1}{T^{\prime}} v_{f}$

where $\Delta P_{e}=P_{e}-P_{m 0}$

$T^{\prime}=\frac{x d s^{\prime}}{x d s} T d o^{\prime}$

$v_{f}=\frac{V s}{x d s} \sin \partial\left[k c u f+T d o^{\prime}\left(x d-x d^{\prime}\right) \frac{V s}{x d s} \sin \partial w\right]+T^{\prime} \frac{V s}{x d s} E q \cos \partial w-P_{m 0}$

$\Delta \dot{\mathrm{P}}_{\mathrm{e}}$ is a linear system with new input $\boldsymbol{v}_{\boldsymbol{f}}$. Robust control techniques for linear systems can be employed. By solving an Algebraic Riccati Equation (ARE), the DFL compensating control law is obtained as

$v_{f}=-K_{\partial} \partial-K_{w} w-K_{P} \Delta P e$

where $\mathrm{K}_{\partial}, \mathrm{K}_{\mathrm{w}}, \mathrm{K}_{\mathrm{P}}$ are linear gains obtained from solution of (ARE).

\subsection{Voltage controller}

Voltage regulation is an important issue particularly in the past-transient period.

$\Delta \dot{V}_{t}=f_{1}(t) w+\frac{f_{2}(t)}{T d o^{\prime}} \Delta P_{e}+\frac{f_{2}(t)}{T d o^{\prime}} v_{f}$

$\mathrm{f}_{1}(\mathrm{t})$ and $\mathrm{f}_{2}(\mathrm{t})$ are highly non-linear functions of $\delta$, Pe and Vt. A new linearized system which is represented by the vector $\left[\Delta \mathrm{Vt}, \mathrm{w}, \Delta \mathrm{P}_{\mathrm{e}}\right]$ can be developed.

Robust linear control techniques can be applied to obtain

$v_{f}=-K_{v} \Delta V t-K_{w} w-K_{P} \Delta P e$

Since voltage is introduced as a feedback variable, the post-fault voltage is prevented from excessive variation.

As the design of voltage controller involves estimating nonlinearity bounds within a certain operating region, it is only effective locally. When a serious disturbance occurs which cause the system to operate in a wider range outside the estimated one, the designed system may not perform well.

To conclude, voltage controller achieves voltage regulation, but it is only valid locally.

\subsection{Co-ordinated control by switching}

It is proposed where a switching strategy is used between the different control actions to ensure transient stability enhancement and voltage regulation.

A typical switching scheme is as follows:

Step 1: when fault occurs at $\mathrm{t}=\mathrm{t}_{0}$, the DFL non-linear controller $\mathrm{u}_{\mathrm{f}}$ with $\boldsymbol{v}_{\boldsymbol{f} \mathbf{1}}$ is employed to maintain transient stability. 
Step 2: At $\mathrm{t}=\mathrm{t}_{\mathrm{s}}$, the control law switches to voltage controller $\mathrm{u}_{\mathrm{f}}$ with $\boldsymbol{v}_{\mathrm{f} \mathbf{2}}$ to maintain desired post-fault voltage level. The switching time $t_{\mathrm{s}}$ should be chosen within the post-transient period, which requires that the fault sequence must be known as a prior. Further the exact switching time has to be determined by trial in simulation.

Thus, as the switching time is physically fixed according to one particular fault sequence, it might not be suitable for different fault and may destabilize the power system. Also the switching time fixed in the first post-transient period cannot achieve over the whole working region allowing for later faults.

The simulation results of different types of controllers which were discussed above have been presented in later section.

\section{Design of a new DFL co-ordinated controller}

We observe that the DFL excitation controller cannot keep the system transiently stable in all fault conditions. Here we concentrate on the design of a nonlinear coordinated controller [15] to overcome the difficulty. By considering the equations and the nonlinear compensating law, we obtain the following linearized plant:

where

$$
\dot{x}(t)=A x(t)+B u(t)
$$

$$
x(t)=\left[\begin{array}{lllll}
\partial(t) & w(t) & P_{e}(t) P_{m}(t) & X_{E}(t)
\end{array}\right]^{T} \text { and } u(t)=\left[v_{f}(t) P_{c}(t)\right]^{T}
$$

Because of the mechanical behaviour of the steam valve, it is expected to come into action only in the transient period. To ensure the stability of the overall system after the power control loop switches off, the feedback control law is structured as follows:

$$
\left[\begin{array}{l}
v_{f}(t) \\
P_{c}(t)
\end{array}\right]=-\left[\begin{array}{lllcc}
k_{\partial 1} & k_{w 1} & k_{e 1} & 0 & 0 \\
k_{\partial 2} & k_{w 2} & k_{e 2} & k_{m 2} & k_{x 2}
\end{array}\right] x(t)
$$

The feedback gain matrix can be obtained by using the following design procedure:

Step 1: Assuming mechanical input power $\mathrm{P}_{\mathrm{m}}(\mathrm{t})$ is constant, use the DFL linearization technique to linearize the plant through the excitation loop. Then use LQ optimal theory to find a feedback control law for the linearized model.

$$
v_{f}(t)=-k_{\partial 1} * \Delta \delta(t)-k_{w 1} * w(t)-k_{e 1} * \Delta P_{e}(t)+P_{m 0}
$$

Step 2: To find the close loop system through the excitation loop. Then use linear quadratic optimal theory to find feedback control law for the linearized model through the power control loop

$$
P_{c}(t)=-k_{\partial 2} * \Delta \delta(t)-k_{w 2} * w(t)-k_{e 2} * \Delta P_{e}(t)-k_{m 2} *\left(P_{m}(t)-P_{m 0}\right)+P_{m 0}-k_{x 2} *\left(X_{E}(t)-X_{E 0}\right)+P_{m 0}
$$

To achieve better post-fault performance of the generator terminal voltage $\mathrm{V}_{\mathrm{t}}(\mathrm{t})$, a DFL variable structure controller is proposed, the control sequence is

Step 1: the fault occurs at $\mathrm{t}=\mathrm{t}_{0}$ and the feedback law is

$$
\begin{gathered}
P_{c}(t)=-k_{\partial 2} * \Delta \delta(t)-k_{w 2} * w(t)-k_{e 2} * \Delta P_{e}(t)-k_{m 2} *\left(P_{m}(t)-P_{m 0}\right)+P_{m 0}-k_{x 2} *\left(X_{E}(t)-X_{E 0}\right)+P_{m 0} \\
v_{f 1}(t)=-k_{\partial 1} * \Delta \delta(t)-k_{w 1} * w(t)-k_{p 1} * \Delta P_{e}(t)+P_{m 0}
\end{gathered}
$$

Both $\mathrm{u}_{\mathrm{f}}(\mathrm{t})$ and $\mathrm{P}_{\mathrm{c}}(\mathrm{t})$ are being used to improve transient stability of the system.

Step 2: at $\mathrm{t}=\mathrm{t}_{1}$ the feedback law through the power control loop switches to

$P_{c}(t)=P_{m 0} \quad$ (because of the mechanical behavior of the turbine, after the system is transiently stable we

remove the governor input( the steam valve control) to reduce the control cost in $P_{c}(t)$.)

Step 3: at $\mathrm{t}=\mathrm{t}_{2}$ the feedback law switches to

$$
v_{f 2}(t)=-k_{v 3} *\left(V_{t}(t)-V_{t 0}\right)-k_{w 3} * w(t)-k_{p 3} * \Delta P_{e}(t)+P_{m 0}
$$

here $\quad P_{c}(t)=P_{m 0} \quad$ whereafter the system is in the post-transient period. The generator terminal voltage $V_{t}(t)$ control (relax the power angle control) is used to improve post fault regulation of the generator terminal voltage $V_{t}(t)$. $t_{1}$ and $t_{2}$ are called the switch time which can be determined by trial and error in simulation $t_{0}$ is obtained from the circuit breaker data.

The new DFL coordinated controller can effectively improve the transient stability of the power system. The system can maintain transient stability even in the case where a large sudden fault occurs close to the generator terminals $(\lambda=0.001)$. The new DFL coordinated controller can achieve better transient stability results than the excitation controller irrespective of the operating point of the system and the fault sequence. 


\section{Simulation results}

In this section, through simulations, results of power angle and terminal voltage responses for different cases have been shown. Fig. 2 and 3 shows the power angle and voltage responses for initial power angle of 72 degree and mechanical input power of 0.9 p.u.

The controllers employed in the simulations as discussed in equation (16) and (18) are:

DFL-LQ controller

Voltage controller

$$
v_{f 1}=19.3 \partial+6.43 w-47.6 \Delta P e+P m o
$$

$$
v_{f 2}=-47.03 \Delta V t+6.93 w-28.6 \Delta P e+P m o
$$

Fig.4 and fig. 5 shows the influence of initial conditions of $\partial$ detector response corresponding to $\partial \mathrm{o}=42,47,52$.

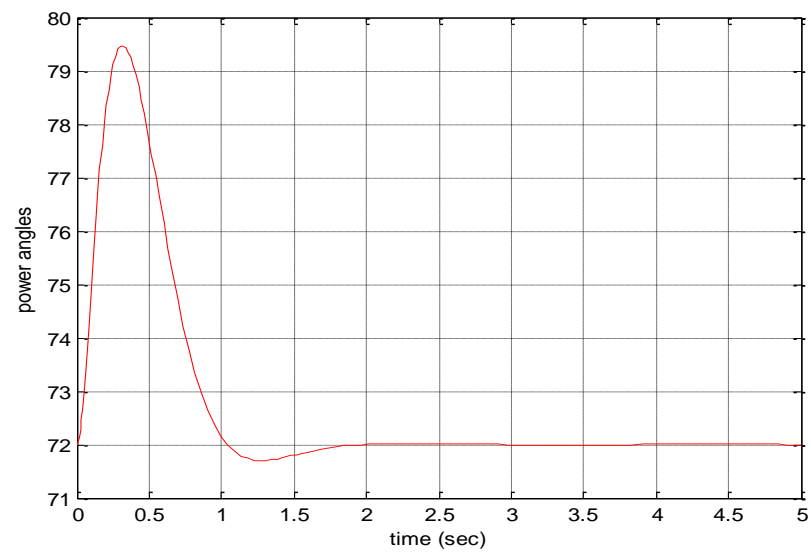

Fig 2: Power angle response for initial angle 72 and Mechanical power, $\mathrm{Pm} 0=0.9$

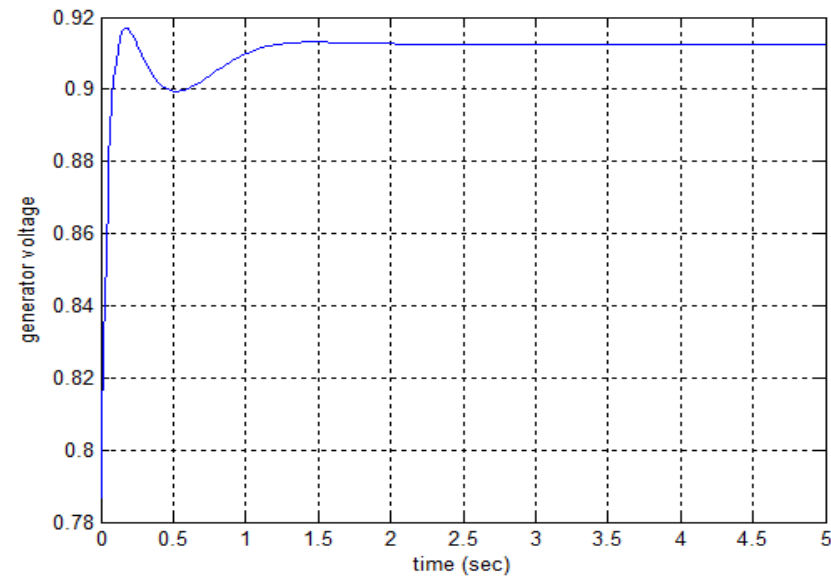

Fig 3: Terminal voltage for initial angle 72 and Mechanical power, Pm0=0.9

It is seen that using only DFL-LQ optimal controller or a DFL voltage regulator, both good transient response and good post-fault performance has not been obtained. So, a switching strategy is used between the different control actions to guarantee transient stability enhancement and voltage regulation.

A typical switching scheme is selected as discussed above. The switching time $t_{s}$ should be reasonably chosen within the post-transient period, which requires that the fault sequence must be known as a prior. Further the exact switching time has to be determined by trial in simulation. The simulation results for co-ordinated controllers are shown in figure 6 and 7. 


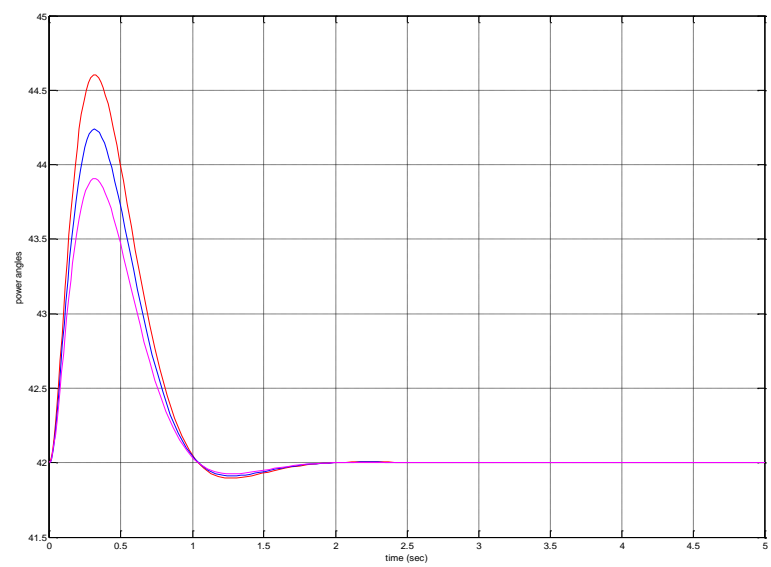

Fig 4: Power angle response for initial angles 42, 47 and 52 and Mechanical power, Pm0=0.45

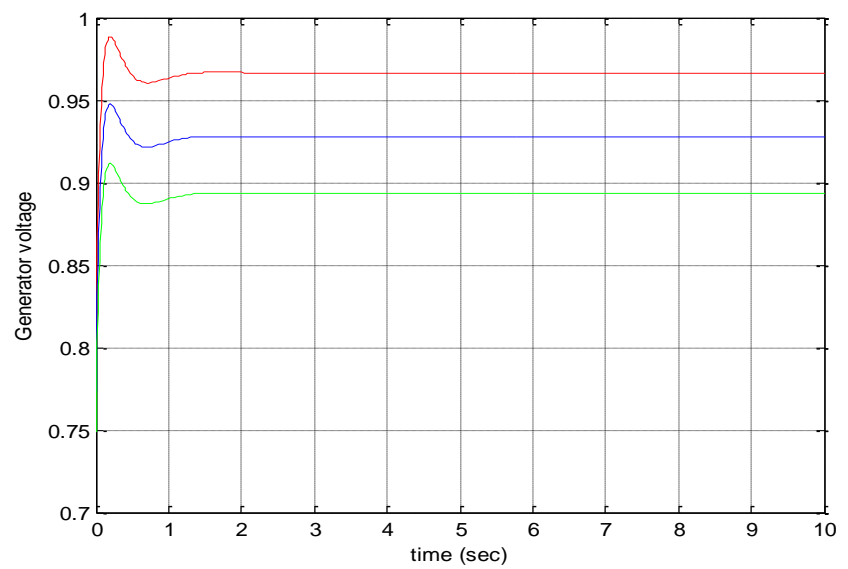

Fig 5: Terminal voltage for initial angles 42, 47 and 52 and Mechanical power, $\mathrm{Pm} 0=0.45$

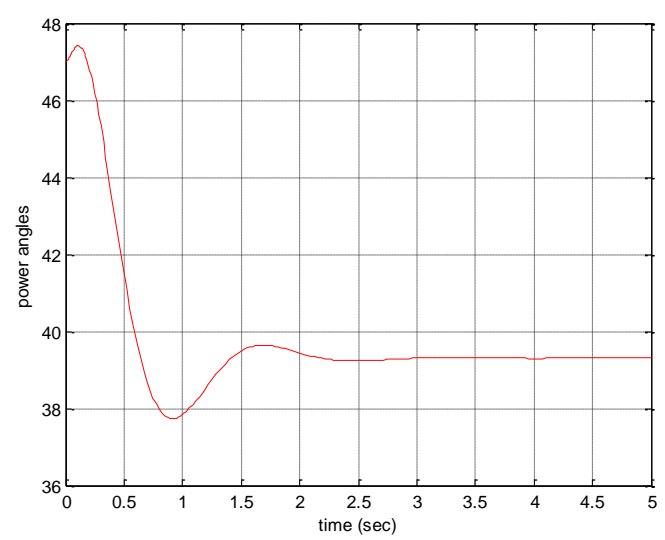

Fig. 6: Power angle response for Excitation controller

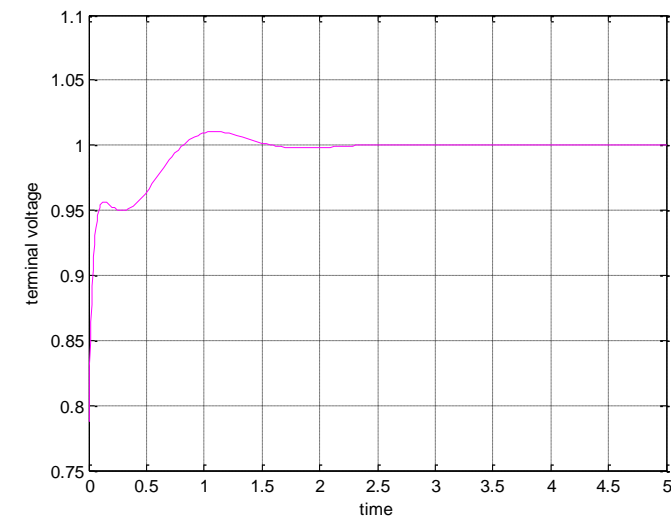

Fig. 7: Voltage response for Excitation controller

\section{Conclusion}

Variable structure excitation and coordinated controllers for a power system have been considered in this paper to achieve both transient stability enhancement and good post-fault performance of the generator terminal voltage $\boldsymbol{V}_{\boldsymbol{t}}(\boldsymbol{t})$. The above technique is an alternative to the usual AVR/PSS combination in generator control. Design procedure has been developed for the controllers. The controllers have been tested through simulation in different cases and the 
performances of the system have been compared. The simulated results show that both transient stability enhancement and good post-fault performance of the generator terminal voltage $\boldsymbol{V}_{\boldsymbol{t}}(\boldsymbol{t})$ have been achieved by using co-ordinated controller as they are independent of the operating point of the system. The coordinated controller maintains transient stability of the system even when a large sudden fault occurs close to the generator terminal and it is also simple and effective. The simulations have been carried out by keeping mechanical power constant. It has been also observed that for a large sudden fault occurring near the generator terminal, the system becomes unstable. The controller can be further improved by considering mechanical power as one of the variable parameter during transient period.

\section{Acknowledgements}

We would sincerely like to thank Professor Kanti B. Datta, Retd. Professor, IIT Kharagpur, for his kind help as without his help it would not have been possible to compile this paper.

\section{References}

[1] Machowski, J.; Bialek, J.W.; Robak, S.; Bumby, J.R.; Excitation control system for use with synchronous generators, IEE Proceedings Generation, Transmission and Distribution, Sep 1998 Vol: 145, p. 537 - 546.

[2] Chapman, J.W.; Ilic, M.D.; King, C.A.; Eng, L.; Kaufman, H.; Stabilizing a multimachine power system via decentralized feedback linearizing excitation control, IEEE Transactions on Power Systems, Aug 1993, Vol. 8 p. 830 - 839.

[3] Bazanella, A.S.; e Silva, A.S.; Kokotovic, P.V.; Lyapunov design of excitation control for synchronous machines, Proceedings of the 36th IEEE Conference on Decision and Control, 1997., p: 211 - 216 vol.1

[4] Gan D.; Qu Z.; Cai H., Multi machine power system excitation control design via theories of feedback linearization control and nonlinear robust control, Int. Journal of Systems Science, Volume 31, Number 4, 1 April 2000 , pp. 519-527.

[5] Sun, C.; Zhao, Z.; Sun, Y.; Lu, Q.; Design of nonlinear robust excitation control for multi machine power systems, IEE ProceedingsGeneration, Transmission and Distribution, May 1996, Vol.143, p.253-257.

[6] Schaefer, R.C.; Excitation control of the synchronous motor, IEEE Transactions on Industry Applications, May/Jun 1999, Vol: 35 Issue: 3

[7] Handschin, E.; Hoffmann, W.; Reyer, F.; Stephanblome, T.; Schlucking, U.; Westermann, D.; Ahmed, S.S.; A new method of excitation control based on fuzzy set theory, : IEEE Transactions on Power systems, Feb 1994, vol.9, p.533-539

[8] Kitauchi, Y.; Taniguchi, H.; Experimental verification of fuzzy excitation control system for multi-machine power system, IEEE Tansaction Energy Conversion, Mar 1997, Vol. 12, p. 94 - 99.

[9] Wang Y, David J Hill, Richard H. Middleton, transient stability enhancement and regulation of power systems, IEEE Trans.onPowerSystems, vol.8,no.2,may1993.

[10] Wei-Der Chang and Shun-Peng Shih, PID controller design of nonlinear systems using an improved particle swarm optimization approach, Jan 2010.

[11] Chao-Chung Peng - Chieh-Li Chen Dynamic controller design for a class of nonlinear uncertain systems subjected to time-varying disturbance, Nonlinear Dyn (2009) 57: 411-423, Nov 2008.

[12] Gao, L, Chen, L, Fan,Y and Ma, HW ‘ A non-linear control design for power systems’ Automatica, vol 28, no.5 (1992) pp. 975-979.

[13] Khargonekar, P.P., I.P.Peterson and K. Zhou, Robust Stabilization of uncertain linear systems: Quadratic Stabilizability and Hळ control theory, IEEE Trans. Auto. Control, AC-35, pp.356-361,1991.

[14] Xie, L., M.Fu and C.E.de Souza, Hœ control and quadratic stabilization of systems with parameter uncertainity via output feedback, IEEE Trans. Auto. Control, 1992.

[15] Wang, Y., L.Gao and D.J.Hill. On the design of new nonlinear excitation controllers of power systems. Proc. of 2nd Int. Conf. Autom., Robotics and Compu. Vision, Singapore, 1992.

[16] Belarbi. "GA for the design of a class fuzzy controller: An alternative approach, IEEE Trans. on Fuzzy Systems 2000, vol.8, Issue- 4, pp. 398405 . 